\title{
Parallel Computing of Graph-based Functions in ReRAM
}

\author{
SAMAN FROEHLICH, Group of Computer Architecture, University of Bremen, Germany \\ SAEIDEH SHIRINZADEH, Fraunhofer Institute for Systems and Innovation Research, Germany \\ ROLF DRECHSLER, Group of Computer Architecture, University of Bremen, Germany and Cyber \\ Physical Systems, DFKI GmbH, Germany
}

Resistive Random Access Memory (ReRAM) is an emerging non-volatile memory technology. Besides its low power consumption and its high scalability, its inherent computation capabilities make ReRAM especially interesting for future computer architectures. Merging computations into the memory is a promising solution for overcoming the memory bottleneck.

To perform computations in ReRAM, efficient synthesis strategies for Boolean functions have to be developed. In this article, we give a thorough presentation of how to employ parallel computing capabilities of ReRAM for the synthesis of functions given state-of-the-art graph-based representations AIGs or BDDs. Additionally, we introduce a new graph-based representation called m-And-Inverter Graph (m-AIGs), which allows us to fully exploit the computing capabilities of ReRAM. In the simulations, we show that our proposed approaches outperform state-of-the art synthesis strategies, and we show the superiority of m-AIGs over the standard AIG representation for ReRAM-based synthesis.

\section{CCS Concepts: • Hardware $\rightarrow$ Memory and dense storage; Logic synthesis;}

Additional Key Words and Phrases: ReRAM, RRAM, in-memory computing, m-AIG, graph-based synthesis, parallel computation

\section{ACM Reference format:}

Saman Froehlich, Saeideh Shirinzadeh, and Rolf Drechsler. 2022. Parallel Computing of Graph-based Functions in ReRAM. f. Emerg. Technol. Comput. Syst. 18, 2, Article 41 (January 2022), 24 pages.

https://doi.org/10.1145/3453163

\section{INTRODUCTION}

As CMOS approaches its physical boundaries due to the continuous shrinking feature size, search for promising emerging technologies beyond scaling limit has sped up to enable further advancement of future low power, leakage free and nanoscale electronic systems. Resistive Random

Preliminary portions of this article appeared in the Proceedings of the 15th IEEE / ACM International Symposium on Nanoscale Architectures (NANOARCH'19) [8] and in the Proceedings of the IEEE International Symposium on Circuits and Systems (ISCAS’2020) [9].

This work was supported by the German Research Foundation (DFG) under contract number DR 287/35-1.

Authors' addresses: S. Froehlich, Group of Computer Architecture, University of Bremen, Bremen, Germany; email: froehlich@uni-bremen.de; S. Shirinzadeh, Fraunhofer Institute for Systems and Innovation Research, Karlsruhe, Germany; email: saeideh.shirinzadeh@isi.fraunhofer.de; R. Drechsler, Group of Computer Architecture, University of Bremen, Bremen, Germany, Cyber Physical Systems, DFKI GmbH, Bremen, Germany; email: drechsle@uni-bremen.de.

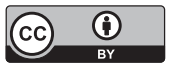

This work is licensed under a Creative Commons Attribution International 4.0 License.

(C) 2022 Association for Computing Machinery.

1550-4832/2022/01-ART41 \$15.00

https://doi.org/10.1145/3453163 
Access Memory (ReRAM) is a non-volatile memory technology that has attracted a high amount of attention among the emerging post-CMOS device technologies thanks to its low power consumption and inherent computing capabilities. These features make ReRAM a promising candidate enabling future computer architectures beyond the classical von Neumann, which suffers from separated memory and processor bottleneck.

Efficiency of logic synthesis on ReRAM crossbars highly depends on the degree of parallelization and the number of required read operations that determine the latency of resulting in-memory implementations. Therefore, maximization of parallel computation and limiting number of memory accesses allowed by the available ReRAM devices/crossbar dimensions are the primary goals of existing synthesis approaches $[2,16]$.

In this article, we propose a comprehensive framework for synthesis and optimization on ReRAM crossbars using logic representations, i.e., Binary Decision Diagram (BDD), conventional two-input And-Inverter graph (AIG). Additionally, we propose an extended form of AIGs, which we call m-And-Inverter Graph (m-AIG). m-AIGs are especially efficient for our presented design methodology. The contributions of this article can be summarized in three main categories based on the selected graph representation as explained in the following.

(1) We present a novel BDD-based synthesis approach for ReRAM crossbars that for the first time utilizes Multiply-Accumulate (MAC) operation instead of logic primitives such as implication [3] or majority function [10] used by related BDD-based approaches [16]. Our proposed approach first optimizes BDDs using an evolutionary algorithm and then directly maps the BDD nodes represented by multiplexers onto parallel MAC operations realized in crossbar columns.

(2) We propose an AIG-based automated compiling procedure for an in-memory computer architecture based on regular ReRAM crossbar arrays. The proposed approach translates arbitrary Boolean functions represented as AIGs into logic-in-memory instruction sets that are executable by applying appropriate voltage levels to bitlines and wordlines of a standard ReRAM crossbar. The compilation procedure allows execution of bit-level parallel computational instructions and lowers the number of required reads considerably. Comparisons with the state-of-the-art show significant improvements with respect to latency and area of the resulting implementations.

(3) We propose a novel graph structure called m-AIG that is at least as compact as classical AIGs. For this graph structure we present a generation procedure, given an AIG representation. Finally, we show how to map them to ReRAM crossbars efficiently and show their superiority compared to traditional AIGs.

This article is supported by extensive simulations, by which we evaluate the proposed design methodologies based on all the three aforementioned graph representations, as well as comparing them with the state of the art. Further, we analyze the efficiency of the propsed m-AIGs and the influence of the parameter $m$ on the results.

\section{RELATED WORK}

This section provides a short study of recent works on synthesis and optimization using ReRAM devices utilizing logic gates and representations similarly to our presented approaches.

BDDs, AIGs, Or-Inverter Graphs, and Majority-Inverter-Graphs (MIGs) have been used for logic synthesis with non-volatile memory devices such as ReRAM in a variety of design methodologies as they allow us to make use of tools and techniques already available for design based on conventional CMOS. In Reference [10], it was shown that the majority of three operation is natively performed with ReRAM. This operation was efficiently used as the basic logic instruction for a 
sequential Programmable in-Memory Architecture (PLiM) based on resistive crossbar arrays. MIGs were therefore used efficiently for synthesis and compilation, as they provide a natural representation of majority-based instruction sequences [19]. Several MIG rewriting algorithms [16] have been adopted by the compiler to reduce the latency of the resulting programs and their required number of memory devices. The compiler only allows a single instruction to be executed per time step and does not count or optimizes the required read operations.

A synthesis approach for a Very Long Instruction Word architecture using crossbar ReRAM called ReVAMP was proposed in Reference [2]. The architecture uses majority operation as the logic primitive similarly to PLiM; however, it supports both read and computational instructions and allows us to execute several computations in parallel under condition of existing shared operands located in the same wordline. In this approach, the maximum possible parallelization is limited by the number of concurrent shared operands and the crossbar dimensions, in particular the number of columns/bitlines.

As discussed above, MIGs have been successfully applied to ReRAM-based architectures mainly using majority operation as the logic primitive. In Reference [11], another logic primitive called Memristor-Aided LoGIC (MAGIC) was utilized in an automated synthesis framework. The MAGIC operation is basically a two-input (also extendable to $n$ inputs) NOR gate consisting of two-input devices and an output device that need to be initialized to certain resistive states before computation. MAGIC provides a stateful logic platform and hence does not require read instructions. The synthesis approach abbreviated to SIMPLE [11] starts with an optimized netlist of NOR gates representing the target Boolean function. The netlist is then mapped onto a memristive crossbar considering location and timing constraints optimally such that the latency is minimized. As this approach uses an exact algorithm, it has very low scalability so that the runtime for functions with fewer than 10 input variables is reported to take several days [25].

MAGIC has also been employed by a heuristic design methodology in Reference [25]. The synthesis approach follows a staircase like style of crossbar implementation aiming to obtain a tradeoff between optimization criteria such as latency, area, layout, and the runtime complexity for larger functions. The presented methodology for the crossbar mapping of operations results in a disperse placement of gates leaving a large number of spare devices.

State-of-the-art for logic synthesis on resistive crossbars also benefit from other data structures such as BDDs. Most BDD-based approaches use implementations of multiplexers based on ReRAM devices that are enabled within several computational steps. The BDD levels are then computed following a sequential or parallel methodology $[6,16]$. A comparison of design preferences corresponding to implementation costs has been presented in Reference [16], where simulations of synthesis approaches based on BDDs, AIGs, and MIGs have been carried out for identical benchmarks sets.

\section{PRELIMINARIES}

\subsection{Graph-based Representations}

Graph-based representations of Boolean functions such as BDDs and AIGs play an important role in synthesis. AIGs are a state-of-the-art structure for synthesis of Boolean functions. Besides AIGs, a commonly used representation for synthesis of functions in ReRAM are MIGs.

3.1.1 AIGs. AIGs [12] are representations of Boolean networks. The edges represent wires between two-input AND gates that correspond to the nodes. Additionally, the edges can be complemented to represent inverters between the nodes. Each terminal node of an AIG corresponds to an input of the Boolean network, while each output node corresponds to an output. Due to their scalability, AIGs play an important role in logic synthesis. 


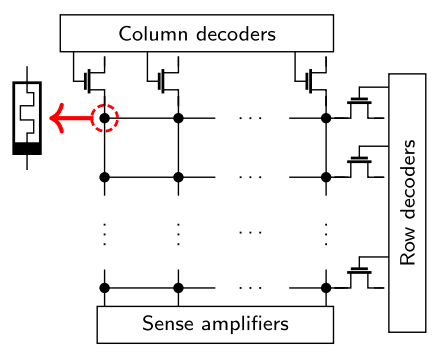

Fig. 1. ReRAM crossbar array.

3.1.2 MIGs. MIGs [1] are a graph-based representation of Boolean functions, where each node represents the three-input majority operation

$$
\langle x, y, z\rangle=(x \wedge y) \vee(x \wedge z) \vee(y \wedge z) .
$$

Edges between nodes represent the connections between majority operations and can be complemented. MIGs contain any AND/OR/Inverter graphs including AIGs and allow for a compact representation.

3.1.3 BDDs. Reduced Order Binary Decision Diagrams, which we call BDDs for brevity, are a graph-based, canonical representation of Boolean functions, consisting of multiple nodes. The nodes are ordered on levels, each level being associated with a Boolean input of the function. We call the number of nodes in a level its level size. Each node $f$ in a BDD has two child nodes $f_{H}$ and $f_{L}$. If the level of $f$ is associated with the variable $x_{i}$, then $f$ implements the function

$$
f=x_{i} \cdot f_{H}+\bar{x}_{i} \cdot f_{L} \text {. }
$$

3.1.4 Manipulation of ReRAM Devices. ReRAM devices are usually ordered in memory crossbar arrays, where each device is connected to a bitline and a wordline. To change the inherent resistive value of a device, a voltage $V$ has to be applied to the corresponding bit- and wordlines.

A memory crossbar array is depicted in Figure 1. The columns correspond to bitlines and the rows to wordlines. To change the resistive state of a single ReRAM device in such an array, the authors of Reference [7] have introduced a $V / 2$ scheme. To set a ReRAM device into a low resistive state, the corresponding bitline is driven to $-V / 2$, and $V / 2$ is applied to the corresponding wordline using voltage drivers. To not change the resistive state of any other ReRAM device in the crossbar array, all other bitlines and wordlines are driven to GND. This way, the voltage difference between the bitline and the wordline of the other devices is below $V$ and thus not high enough to change their state. Putting a device into a high resistive state can be done in a similar fashion by applying $V / 2$ to the bitline and $-V / 2$ to the wordline. $V / 2$ and $-V / 2$ can be interpreted as logic values 1 and 0 , respectively.

3.1.5 Resistive Majority Operation $\mathrm{RM}_{3}$. A single ReRAM device can be seen as a two-terminal device with the terminals $P$ and $Q$ and the internal resistance state $Z$. A low-resistance state is identified with logic value 1 , while a high-resistance state is identified with logic value 0 . To perform a resistive majority operation, logic values are applied as voltages to $\mathrm{P}$ and $\mathrm{Q}$. This results in the computation of $\mathrm{RM}_{3}(P, Q, Z)=\langle P, \bar{Q}, Z\rangle$ and the result being stored as the new resistive value $Z_{\text {res }}=\mathrm{RM}_{3}(P, Q, Z)$ of the same device (cf. References $\left.[10,13,15]\right)$. When placed in a crossbar array, the bitline is connected to $Q$ and the wordline is connected to $P$. The device performing the computation is called host device. 


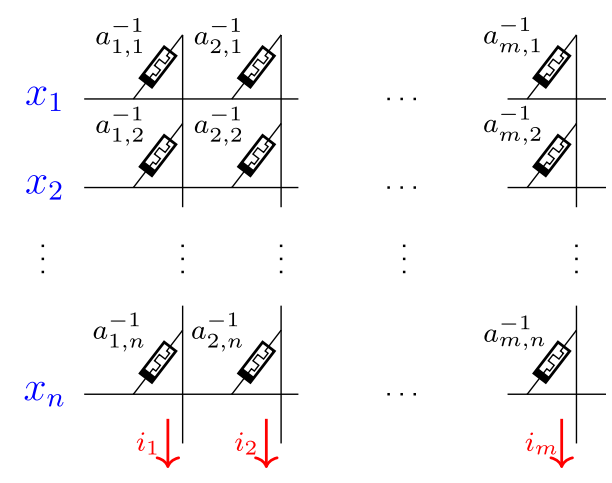

Fig. 2. MAC computation with ReRAM.

Being able to implement $\mathrm{RM}_{3}$ allows ReRAM crossbar arrays to compute any given function, since the $\mathrm{RM}_{3}$ operation is universal. Of particular importance is the direct assignment of the value $X$ to a ReRAM device $Y$ and the inversion of $X$ into $Y$. Both can be performed by two $\mathrm{RM}_{3}$ operations, respectively:

\begin{tabular}{|ll||ll|}
\hline \multicolumn{2}{|c||}{ Inversion } & \multicolumn{2}{c|}{ Assignment } \\
\hline$Y \leftarrow \mathrm{RM}_{3}(1,0, Y)$ & $/ / Y=1$ & $Y \leftarrow \mathrm{RM}_{3}(1,0, Y)$ & $/ / Y=1$ \\
$Y \leftarrow \mathrm{RM}_{3}(0, X, Y)$ & $/ / Y=\bar{X}$ & $Y \leftarrow \mathrm{RM}_{3}(X, 1, Y)$ & $/ / Y=X$ \\
\hline
\end{tabular}

\subsection{ReRAM MAC Computation}

For applications such as neural networks and neuromorphic computing that perform a lot of matrix multiplications, an efficient implementation of the MAC operation is needed. ReRAM provides the basis for such an efficient implementation, by allowing us to compute multiple MAC operations in parallel.

Consider the ReRAM crossbar depicted in Figure 2. The ReRAM crossbar in this figure computes $I=A x$, where $I=\left(i_{1}, \ldots, i_{m}\right)^{T}, x=\left(x_{1}, \ldots, x_{n}\right)^{T}$ and

$$
A=\left(\begin{array}{ccc}
a_{1,1} & \ldots & a_{1, n} \\
\vdots & \ddots & \vdots \\
a_{m, 1} & \ldots & a_{m, n}
\end{array}\right) .
$$

The resistive values of the ReRAM devices are set to $a_{j, k}^{-1}$. Applying the voltages $x_{1}, \ldots, x_{n}$ to the corresponding rows computes $i_{j}=\sum_{k=1}^{n} a_{j, k} \cdot x_{k}$. Thus, after writing the resistive values $a_{j, k}^{-1}$ to the corresponding devices, $m$ MAC operations are computed in parallel within a single cycle, each consisting of $n$ multiplications.

\section{GRAPH-BASED COMPUTATION IN RERAM}

To perform graph-based computations of Boolean functions in ReRAM, existing approaches have tried to find efficient ways of mapping nodes to the ReRAM devices [16]. Since often MIGs need the smallest number of operations and devices, they have become the state-of-the art graph structure for ReRAM-based synthesis. However, existing approaches do neither focus on parallel computations, nor do they use the MAC operation to perform the evaluation but rely on logic primitives. While we argue why this is not efficient for MIGs in this section, we show how to perform parallel 


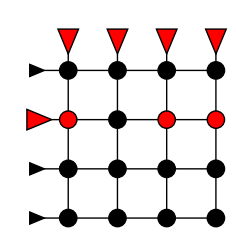

(a) Wordline

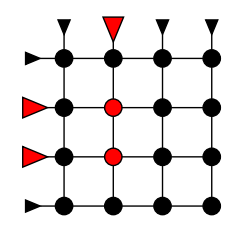

(b) Bitline

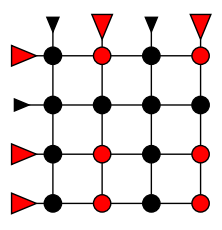

(c) Mixed

Fig. 3. Examples for three kinds of parallel computation. Active devices are highlighted in red.

computation of multiple AIG nodes, which yields a significant performance boost compared to state-of-the art methods. Additionally, we show how to use the MAC operation to perform parallel computations for BDD-based synthesis reducing the overall costs for BDD-based synthesis on ReRAM.

\subsection{Types of Parallelism}

Parallel computations may be performed on a ReRAM array by addressing multiple wordlines or bitlines simultaneously. We say that a computation is wordline parallel if it uses one wordline and multiple bitlines; we call it bitline parallel if it uses one bitline and multiple wordlines; and we call it mixed parallel if it uses multiple wordlines and multiple bitlines.

Figure 3 shows an example for each type of parallelism. Row and column drivers are represented by triangles; active devices are shown in red and slightly enlarged. The ReRAM devices in which a computation takes place are highlighted in red.

Mixed parallel computation has to deal with data distortion; even the small computation in Figure 3(c) cannot be performed if only one of the six activated devices contains a value that must not be overwritten. Since the problem becomes more severe as more lines are activated, it is unfeasible to make efficient use of this parallelism in general when performing $\mathrm{RM}_{3}$-based computations.

Bitline parallel computation cannot deal with inversions efficiently, which are central to most logic representations. This is caused by the fact that in $\mathrm{RM}_{3}$-based computations, the bitline is naturally inverted (cf. Section 3.1.5), but the wordline and the internal resistance state are not. Thus, computing inversions in a bitline parallel design requires significant overhead.

While wordline parallel computation cannot directly parallelize assignment operations, it can parallelize inversion. Assignment can then be realized by double inversion. Since it offers the most versatility, we focus on enabling wordline parallelism in this article.

\subsection{BDD-based Parallel Computation of Boolean Function in ReRAM}

We propose an approach for logic synthesis on regular ReRAM crossbars that uses BDDs for efficient representation of arbitrary Boolean functions. The proposed approach particularly benefits from efficient realization of logic primitives, i.e., multiplexers in case of BDDs, using the MAC operation executed in ReRAM crossbar columns. This section explains the procedure to compute a BDD on ReRAM crossbar and discusses the cost functions and employed optimization schemes to tackle them.

To compute a BDD on a ReRAM crossbar, every node has to be realized as a $2 \times 1$ multiplexer designating Boolean relation $\bar{s} \cdot x+s \cdot y$, where $s$ is the select line and $x$ and $y$ are the two inputs. For BDD implementation, all multiplexers realizing nodes in the $i$ th BDD level use an identical input variable of the target function as select line. In a BDD representing a function with $N$ input variables using an initial ascending variable ordering, i.e., $x_{0}<x_{1}<\cdots<x_{(N-1)}$, level $i$ corresponds 


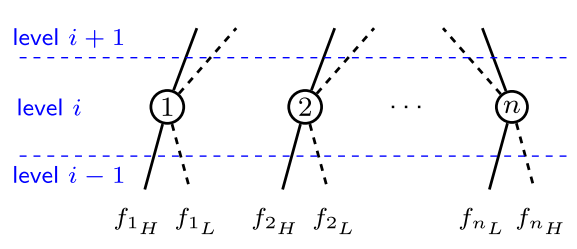

(a)
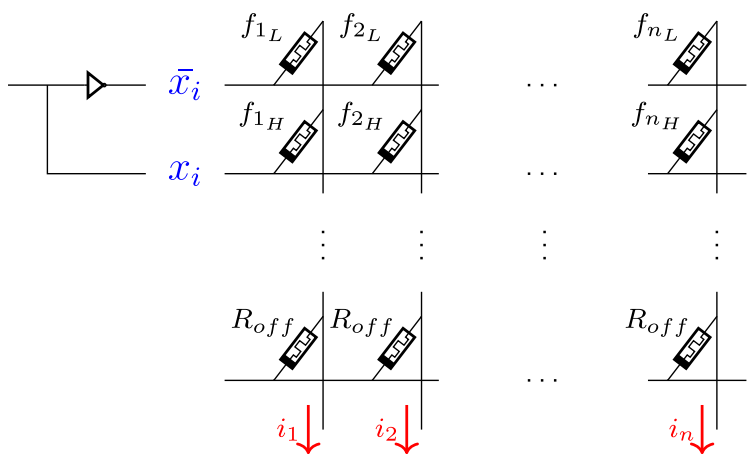

Fig. 4. BDD level computation on ReRAM crossbar using MAC operation.

to input variable $x_{i}$. Using an arbitrary variable ordering, a BDD level of index $i$ can correspond to any input variable with a different index $j$, while $j \in 0, \ldots, N-1$.

Assume the BDD level shown in Figure 4(a). Level $i$ including $n$ nodes is eligible for computation when the low and high child nodes, i.e, $f_{i_{L}}, f_{i_{H}}, 1 \leq i \leq n$ are previously computed. In this case, each node can be computed by a single MAC operation while the low and high child nodes are stored in two successive rows within the same column as shown in Figure 4(b). The output of MAC operation at each column under assumption that the rest of ReRAM devices are under zero voltage and ideally do not pass any electrical current is equal to $\bar{x}_{i} \cdot f_{i_{L}}+x_{i} \cdot f_{i_{H}}$, representing the value of node $i$.

Using the MAC operation as explained above, a BDD can be computed on a ReRAM crossbar starting from the bottom of the graph, i.e., 0 and 1 terminals. To compute each level, first the low and high child nodes have to be stored in the first and second row of the crossbar, respectively. Assuming that one word with a maximum length of $r$ can be written into a row at each cycle, for a BDD level with a size of $n_{l}$, i.e., number of nodes, it takes $2\left\lceil\frac{n_{l}}{r}\right\rceil$ write cycles to store the child node values on two crossbar rows. For example, for a level with 18 nodes while using a write register with 16 bits it takes 4 write cycles to have the level inputs ready on the ReRAM, as both low and high child nodes' values exceed the register size.

When the inputs of the BDD level are stored as shown in Figure 4(b), the MAC operation can be carried out by applying the input variable corresponding to the level being computed. After computation, the currents of the columns are fed into Analog to Digital Converters and then written into the crossbar in resistance form to be used as the inputs of the next level. This procedure continues until all levels are computed.

The number of writes to the memory in the approach presented above depends on BDD level sizes, the write register length, and nonconsecutive fanouts/BDD node outputs that target levels not immediately after their origin level. Figure 5(a) shows a BDD-based on initial ordering that has a nonconsecutive fanout indicated in red targeting the second level, while originating from level four. Since the presented synthesis procedure uses the outputs of the nodes in the very next level without permanent copies, such fanouts have to be copied to be used at the fanout targets. The copy devices can be located nearby node devices in the remaining idle columns as shown in Figure 5(b). In this case, the total number of write cycles required to compute a BDD on ReRAM with our proposed approach is equal to

$$
O P=\sum\left\lceil\frac{n_{l}}{r}\right\rceil \cdot 2+\sum\left\lceil\frac{f_{l}}{r}\right\rceil
$$




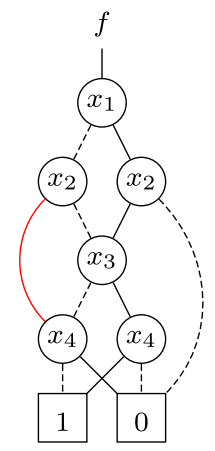

(a)

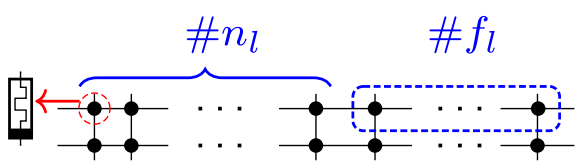

(b)

Fig. 5. (a) Example BDD with a nonconsecutive fanout indicated in red. (b) Storing nodes and nonconsecutive fanout values in ReRAM crossbar.

where $n_{l}$ is the level size, $r$ is the length of the write register, and $f_{l}$ is the number of nonconsecutive fanouts in the entire graph.

The devices for node computations are reused for the next level, and therefore their total number depends on the maximum level size. However, the copy devices for nonconsecutive fanouts are not reused during implementation, so they are summed. Accordingly, the total number of ReRAM devices required is as follows:

$$
R=\left(\max \left\lceil\frac{n_{l}}{r}\right\rceil \cdot 2+\sum\left\lceil\frac{f_{l}}{r}\right\rceil\right) \cdot r .
$$

An optimization approach has been developed that targets the number of write cycles and ReRAM devices needed by the proposed synthesis approach that are defined above. For this purpose, we use a prioritized- $\varepsilon$-preferred EA [17] for variable ordering that has been efficiently applied to BDDs. We consider the latency a more important criterion and give a higher priority to it compared to the number of devices representing area.

As discussed, both of the cost functions representing latency and area of the resulting implementations depend on the level sizes and number of nonconsecutive fanouts denoted by $n_{l}$ and $f_{l}$, respectively. Indeed, a BDD with smaller levels and fewer nonconsecutive fanouts can be computed more efficiently on ReRAM crossbar, requiring a smaller number of write cycles and devices. Therefore, maximum value of $n_{l}$ over all BDD levels as well as $f_{l}$ have been considered as optimization criteria in a third priority level.

\subsection{AIG-based Parallel Computation of Boolean Function in ReRAM}

4.3.1 Enabling Parallelism. Synthesis for $\mathrm{RM}_{3}$-based in-memory computing has made ample use of MIGs for both sequential as well as parallel architectures $[2,19]$. When computing two MIG-nodes in parallel within a wordline we have to respect four constraints:

(1) All children of both nodes must be computed. In particular, there must not be any data dependencies between the nodes.

(2) The nodes must share a wordline operand.

(3) The host devices of the nodes must be placed in the same wordline.

(4) Since it is overwritten, the content of the host devices must not be necessary for any other computations. 


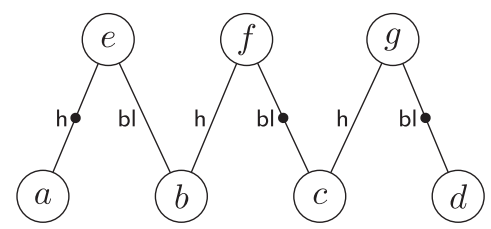

(a)

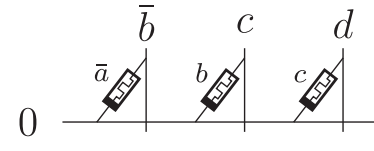

(b)

Fig. 6. (a) Example AIG-level. (b) Mapping to a ReRAM crossbar.

While the first constraint can be dealt with by levelization, the last three constraints severely hinder parallelization efforts for general MIGs.

Every AIG can be represented as an MIG where each node has a constant zero child. This is an ideal situation for parallelization, since it completely alleviates Constraint 2 . In contrast to previous approaches, we therefore propose to use AIGs instead of MIGs as logic representation of parallel logic computation. When computing an AIG node, we always apply logical zero to the wordline.

Each node of the AIG is associated with a ReRAM device on the crossbar that holds its content. To avoid initialization of a new device, previous approaches tried to reuse these devices as hosts to a computation. However, this reuse introduces sequential dependencies even within one level of an AIG in the form of Constraint 4. Moreover, the placement of a single node may determine the placement of nodes in several later levels that inherit its position. This inheritance obstructs satisfaction of Constraint 3. In general, while reusing devices as hosts is important to reduce copy operations, it is detrimental to enabling parallelism. Therefore, we abandon such reuse altogether. Instead, we allocate a new device for each computation and initialize it with the necessary host value. In this way, we have full control over the placement of all computations and alleviate Constraint 4 completely.

This comes at the cost of extra work, since we need to transfer the data of each host node into a new ReRAM device. As was explained in Section 4.1, multiple inversions can be performed in parallel within a wordline. Therefore, we use inversion as initialization operation for AIGs.

Example 4.1. An example is given in Figure 6. Figure 6(a) shows an AIG. In this example, we assume that the nodes $\mathrm{a}, \mathrm{b}, \mathrm{c}$, and $\mathrm{d}$ and their corresponding inverse are already computed, and we want to compute the nodes e, $\mathrm{f}$, and $\mathrm{g}$. Since, in contrast to MIGs, each node of the AIG has only two outgoing edges but the $\mathrm{RM}_{3}$ operation is a ternary operation, we can chose one of the inputs of the $\mathrm{RM}_{3}$ operation to be equal for all devices. To compute the $A N D$ function, we choose the wordline operand to be 0 , since $\operatorname{AND}\left(x_{1}, x_{2}\right)=\mathrm{RM}_{3}\left(x_{1}, \overline{x_{2}}, 0\right)$. Thus, we can place the nodes e, $\mathrm{f}$, and $\mathrm{g}$ on the same wordline and load the value of one of their child nodes into the corresponding host devices. This is depicted in Figure 6(b), where the three devices correspond to the nodes e, f, and g, respectively (i.e., the first device corresponds to node e, the second to node $f$, and the third to node g). After loading the host operands $\bar{a}, b$, and $c$ into the devices, we can compute the nodes $\mathrm{e}, \mathrm{f}$, and $\mathrm{g}$ in parallel by applying 0 to the wordline and the corresponding bitline operands $\bar{b}, \mathrm{c}$, and $\mathrm{d}$ to the bitlines.

4.3.2 Host and Bitline Operands. Each AIG node has two outgoing, possibly complemented edges. One of them will be used as host operand, i.e., loaded into the ReRAM device that will perform the computation, and one will be used as bitline operand, i.e., applied as a voltage to the bitline. Since we use inversion as initialization operation, complemented edges are preferred as host operands. However, since resistive majority naturally inverts its bitline operand, it is beneficial for the second operand to be inverted as well; ideally all operands are inverted. Since this cannot be assumed for a general AIG, we need to perform extra computations to obtain the inverted 


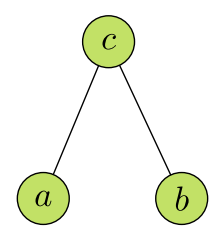

(a)

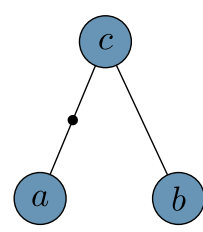

(b)

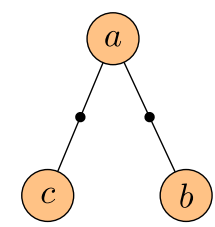

(c)

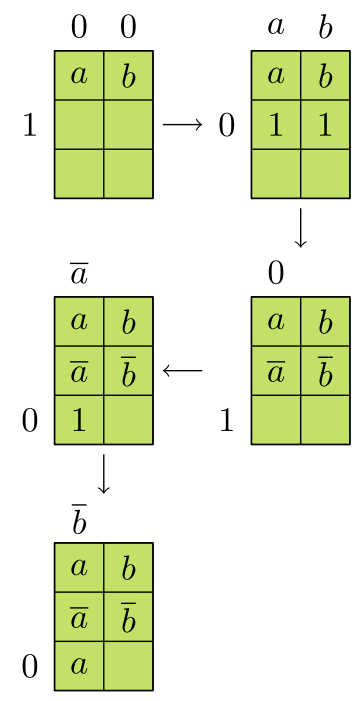

(d)

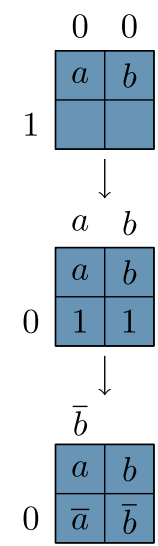

(e)

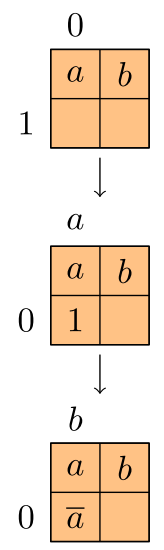

(f)

Fig. 7. Computation with cases for complemented edges.

values. Fortunately, these computations are inversions and can therefore be parallelized efficiently. Moreover, each inverse value needs to be computed at most once.

The decision that outgoing edge is used as host operand and that is used as bitline operand does not affect the number of computations but only the number of reads. As we focus on computations in this article, we simply make this decision before any instruction is generated. The decision is made in an arbitrary fashion, as we are faced with a symmetric situation in which both host and bitline operands need to be read out, simple heuristics do not yield any benefit.

Example 4.2. Figure 7(a)-(c) show the different cases of complemented edges: No outgoing edge is complemented (Figure 7(a), green), one edge is complemented (Figure 7(b), blue), or both edges are complemented (Figure 7(c), orange). If we assume that nodes a and b are already computed and we want to compute node $\mathrm{c}$, then the corresponding computation schemes can be seen in Figure 7(d)-(f). Each grid corresponds to one computational cycle, and each cell in a grid corresponds to one ReRAM device in a crossbar. Wordline and bitline operands are shown to the left and on top of the grids, respectively. The final output (i.e., node c) is stored in the bottom left cell after the final computation. Note, for clarity, that we have not shown the reads in these figures, and we assume that the values of the nodes a and b must not be overwritten.

We can see that on the left-hand side (Figure 7(d), green) where no incoming edges are complemented, overall five devices and four computational cycles are needed. This is caused by the fact that we have to perform a double inversion for the host and the bitline operand of the device in 


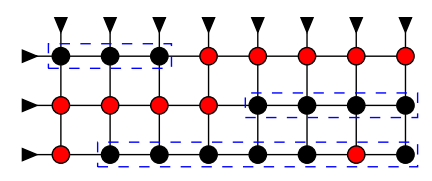

Fig. 8. Crossbar with holes.

which node $\mathrm{c}$ is to be computed. An example where only one outgoing edge is inverted is shown in the middle (Figure 7(e), blue). Here, four devices and three computational cycles are needed. Finally, on the right-hand side (Figure 7(f), orange) an example is shown where both outgoing edges are complemented. This example only requires three devices and three computational cycles for its computation. In conclusion, we can see that in the worst case (Figure 7(a) and (d)), where no outgoing edges are complemented, more computational cycles and devices are needed compared to the other two examples. As soon as one outgoing edge is complemented, the number of computational cycles and the number of needed devices is reduced. When both edges are complemented, additionally the number of devices is reduced even further; however, the number of computational cycles is not.

4.3.3 Managing Allocations. We wish to keep the number of required ReRAM devices as low as efficient parallelism allows. Thus, we keep track of whether the content of a device is still needed and free the device if possible. We call the set of free devices in a word a hole. The devices in a hole can be reused instead of allocating new ones. However, filling a small hole in a word is computationally inefficient, since we forsake the opportunity to perform the computations in parallel with others. Therefore, we introduce a parameter $h$ to the compilation; we try to only reuse holes that are not smaller than $h$.

Example 4.3. An example is given in Figure 8. Here, a schematic ReRAM crossbar with wordsize eight is shown. Active devices are depicted as red circles and slightly enlarged, while free devices are shown as black circles. We can see that the first wordline (i.e., the first row) has a hole size of three, the second wordline has a hole size of four, and the third wordline has a hole size of six. The holes are marked in dashed, blue rectangles. The third wordline shows that the devices of a hole can be spread over a whole wordline (i.e., the unused devices do not have to be adjacent to each other).

4.3.4 Placing Nodes. Each node in our logic representation (and potentially its inverse value) must be assigned to a ReRAM device on the crossbar once it is to be computed. These placements majorly influence the efficiency of the computation; all nodes placed in the same word may be computed in parallel. Moreover, by placing nodes in the same word whose host operands reside on the same wordline we reduce the read cost for initializations, since their host operands can be read simultaneously.

Assume we are given a set $N$ of nodes to be placed and further assume that their children have already been placed on the crossbar. We split $N$ into equivalence classes $N_{1}, \ldots, N_{n}$, where all members of $N_{k}$ have host inputs that reside on the same wordline. These are then again split into groups of size at most $r$. Next, we inspect holes on the crossbar. If a hole is larger than $h$, then we try to fill it. A hole is filled greedily with fitting groups, where larger groups are considered before smaller ones. All groups that remain are placed on newly allocated words (with the same filling algorithm).

We assume that the Primary Inputs (PIs) to the function we compute are present in the memory array that we are computing on. As we never reuse a ReRAM device as host, computation of a function does not destroy its inputs. Therefore, PIs do not have to be copied. However, if they 


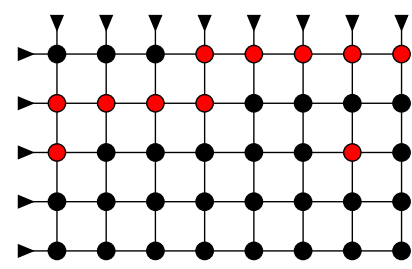

(a) Initial Crossbar

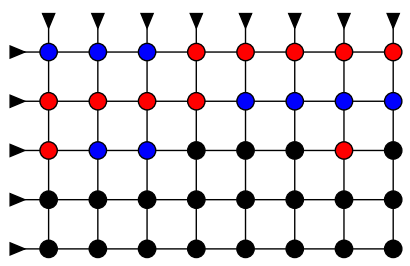

(b) Placing nine nodes with $h=3$

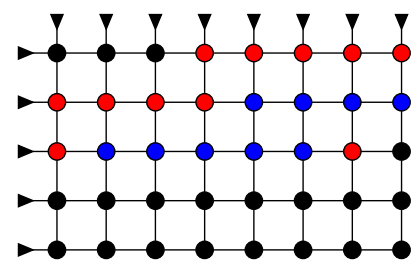

(c) Placing nine nodes with $h=4$

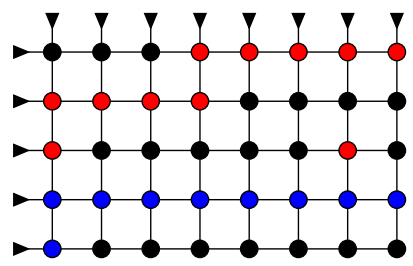

(d) Placing nine nodes with $h=7$

Fig. 9. Examples for hole filling.

are not tightly packed into words, then the number of reads may increase, since the inputs cannot be read in parallel. It may then be beneficial to rearrange them into words. Assuming the worst case, in which each input must be read sequentially, the cost for this operation is $I+5\left\lceil\frac{I}{r}\right\rceil$, where $I$ is the number of PIs; we need to spend one array access on reading each input as well as four computations and one read per $r$ many inputs on double inversions.

This preprocessing step is superfluous when processing wordlevel data, in particular with arithmetic functions. The output level of a function can be written into words again without additional cost, allowing us to chain multiple wordlevel functions without data movement.

Example 4.4. An example for the placement of nodes is given in Figure 9(a)-(d). Figure 9(a) shows a schematic ReRAM crossbar with wordsize eight and the same device allocation as in Example 4.3 in Section 4.3.3. However, the given crossbar has two additional wordlines, which are not yet used. In our example, we consider an equivalence class with nine nodes to be placed. Figure 9(b) shows the placement if $h$ is set to three, where placed nodes are marked in blue. The holes in the first two wordlines are filled. Additionally, two nodes of the third wordline are filled, and thus we need to perform three computational cycles to compute all placed nodes, since three wordlines are used. However, if $h$ is set to four, then the hole in the first wordline is too small to be used. Thus, only the holes in the second and third wordlines are used for placment. This is shown in Figure 9(c), where two computations need to be performed to compute the filled nodes. However, if we choose $h$ to be seven as shown in Figure 9(d), then none of the existing holes can be filled. Thus, two new wordlines need to be allocated.

4.3.5 Computing an AIG. The AIG levels are computed successively by following three steps for each one. First, place and compute the inverted values necessary for this level. Second, place the nodes of the level and initialize the devices with the corresponding host operands. Third, compute the nodes themselves by scheduling the computations that have host devices in the same word in parallel. Reads are scheduled as required by the computations.

Example 4.5. We demonstrate the computation of an AIG level with an example. Consider the AIG in Figure 10. We compute the upper level. To the left of each edge it is indicated whether the edge is used as host operand $(\mathrm{h})$ or as bitline operand (bl). Figure 11 illustrates the computation on 


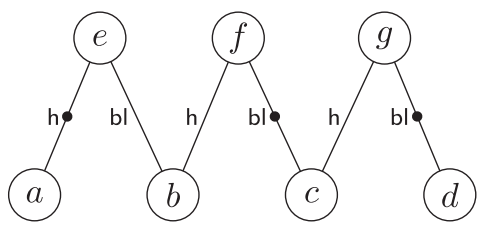

Fig. 10. Example AIG.

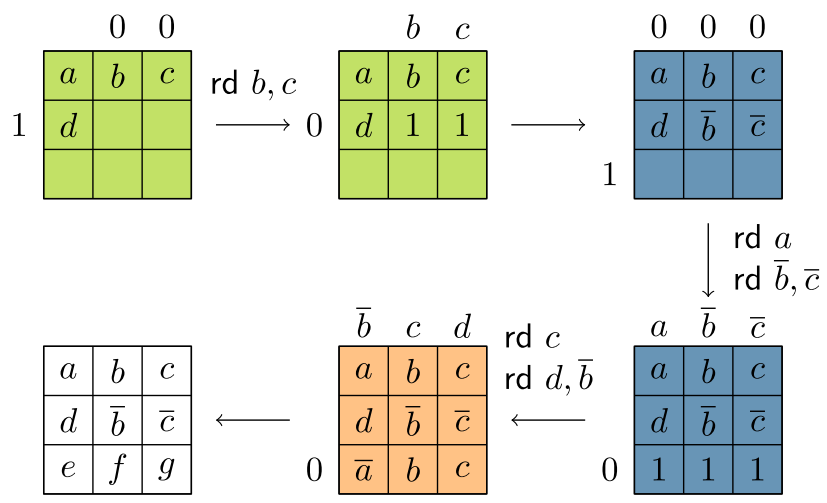

Fig. 11. Computation of the function in Figure 10.

a $3 \times 3$ ReRAM array by displaying the six intermediate states. Operands applied to bitlines and wordlines are indicated outside of the array. We start the computation on the top left, where the lower level of Figure 10 is already present. The three steps discussed in Section 4.3.5 are indicated by the background colours green, blue, and orange, respectively.

Step 1: Compute inverted values (green):

The inverted values necessary for this level are $\bar{b}$ and $\bar{c}$. They are to be placed in the second word. Logical one is written to the respective devices. Then we read out (rd) the values of $b$ and $c$ from the first word to write their inverses.

Step 2: Initialize host devices (blue):

The three nodes to be computed are to be placed in the third word, to which we write logical one. Then we read out the values of $a, \bar{b}$, and $\bar{c}$ before applying them to the bitlines. Since the operands are spread across two words, we need to spend two read operations.

Step 3: Compute level (orange):

The bitline operands $\bar{b}, c$, and $d$ are applied to the bitlines.

\section{M-AND-INVERTER GRAPHS}

In this section, we introduce our novel graph structure m-AIG. While AIGs and BDDs as used in Section 4 are well known and state-of-the art graph structures for the representation of Boolean functions, the strucutre of m-AIGs is tailored for ReRAM devices. m-AIGs exploit the efficient computation capabilities of ReRAM devices, while having at most as many nodes as standard AIGs plus one, yielding an efficient overall representation. We first give an introduction to the structure of $\mathrm{m}$-AIGs in Section 5.1. Subsequently, in Section 5.2 we show how to generate m-AIGs for a 


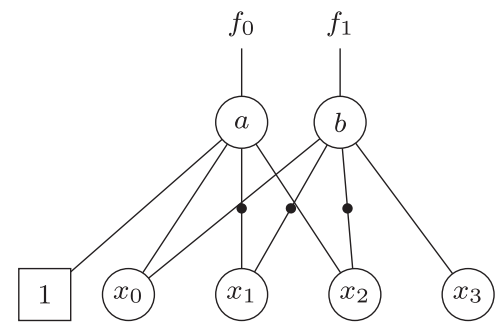

Fig. 12. m-AIG example.

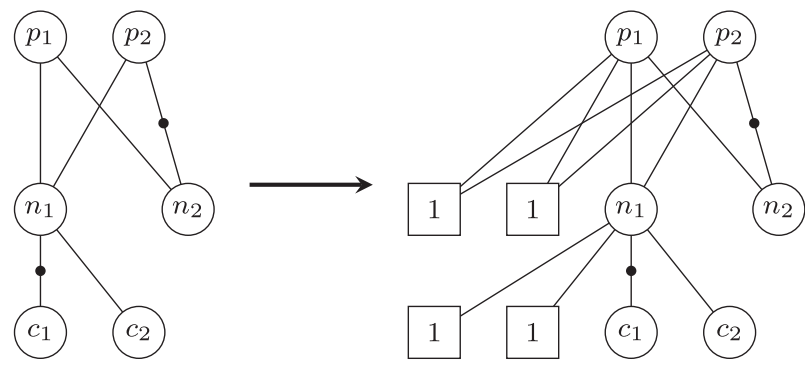

Fig. 13. First step: AIG to m-AIG conversion.

given AIG representation. Finally, in Section 5.3 we present how to map an m-AIG representation to ReRAM devices.

\subsection{Structure of $\mathrm{m}$-AIGS}

m-AIGs are graph-based representations of Boolean functions, where each node represents an mInput And Gate or a PI. Each input edge can either be connected to the constant 1 or to a child node. If the input edge is connected to a node, then the edge may be complemented to indicate inversion. Since AIGs are a subset of m-AIGs, m-AIGs are not canonical.

An example is given in Figure 12, which shows an m-AIG with four PIs $\left\{x_{0}, \ldots, x_{3}\right\}$ and two primary outputs $\left\{f_{0}, f_{1}\right\}$. In this example, $m$ is set to four, and thus each node represents the four input AND operation. However, the constant 1 can be used as input, allowing each node to effectively implement an AND operation with fewer inputs. Here, node $a$ actually implements the three-input AND operation.

\subsection{Generation of $\mathrm{m}$-AIGS}

In this section, we show how to generate an m-AIG for a given AIG representation of a Boolean function. The generation of an $\mathrm{m}-\mathrm{AIG}$ can be seperated into two steps:

(1) In the first step, we add $m$-2 inputs to each node that is not a PI of the AIG, where each additional input is connected to the constant 1 .

(2) After adding the inputs to each node, we simplify the graph using a bottom-up approach in the second step by merging nodes.

The first step is a formal conversion of an AIG into an m-AIG. After this step, the resulting m-AIG has as many nodes as the initial AIG plus one additional node for the constant 1 .

Example 5.1. An example for the first step is given in Figure 13. On the left-hand side, we can see an AIG with six nodes, of which three are no PIs. On the right-hand side, we can see its extension 


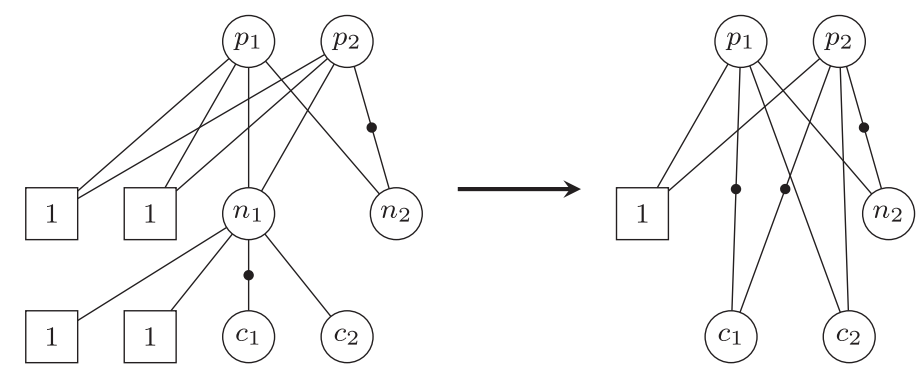

Fig. 14. Second step: m-AIG simplification.

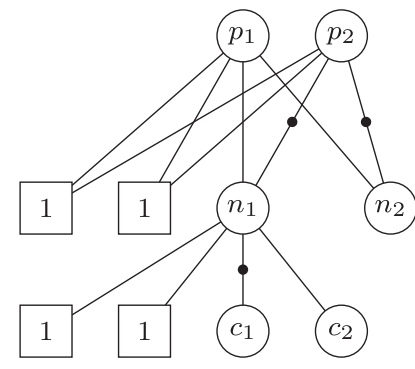

(a)

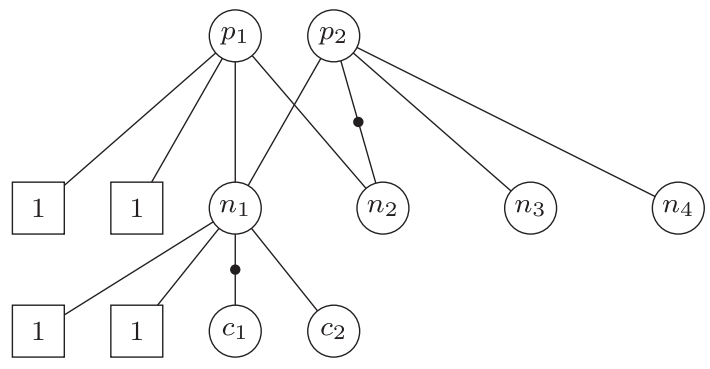

(b)

Fig. 15. No simplification possible.

to an $\mathrm{m}$-AIG with $m=4$, where each node that is no PI is connected to $m-2=2$ constant 1 nodes. It should be noted that all constant 1 nodes would be merged in a real scenario; however, we have depicted them here separately for clarity.

The second step allows us to reduce the total number of nodes in the m-AIG. Our approach is to start from the PIs of the graph and traverse the nodes upwards (i.e., from the PIs to the POs). For each node $n$, we check if the node has only non-complemented edges to its parents. Additionally, for each parent, it is checked if the parent node has at least as many edges to the constant 1 node, as the node $n$ has edges to nodes, which are not the constant 1 decreased by one. This condition ensures that the parent nodes can have edges to all children of the current node $n$. If that is the case for all parents, then the node $n$ can be removed and replaced by its children. In terms of complementation, the edges from the parents to their new children are equal to the edges that were connected to the node $n$. Since this second step can only reduce the number of nodes, the final m-AIG will have at most as many nodes as the original AIG plus one node for the constant 1 .

Example 5.2. An example for the second step is given in Figure 14. Here, on the left-hand side, node $n_{1}$ can be removed, since both edges to its parents $p_{1}$ and $p_{2}$ are not complemented. The simplified graph can be seen on the right-hand side. $p_{1}$ and $p_{2}$ are now directly connected to the children $c_{1}$ and $c_{2}$ of $n_{1}$. The edges to $c_{1}$ are complemented, since the edge from $n_{1}$ to $c_{1}$ was also complemented.

Example 5.3. Figure 15 shows two examples where no simplification is possible. In Figure 15(a), no simplification is possible, since the edge from $n_{1}$ to $p_{2}$ is complemented. Note that even if the edge from $p_{1}$ to $n_{1}$ was complemented additionally, still no simplification would be possible, 


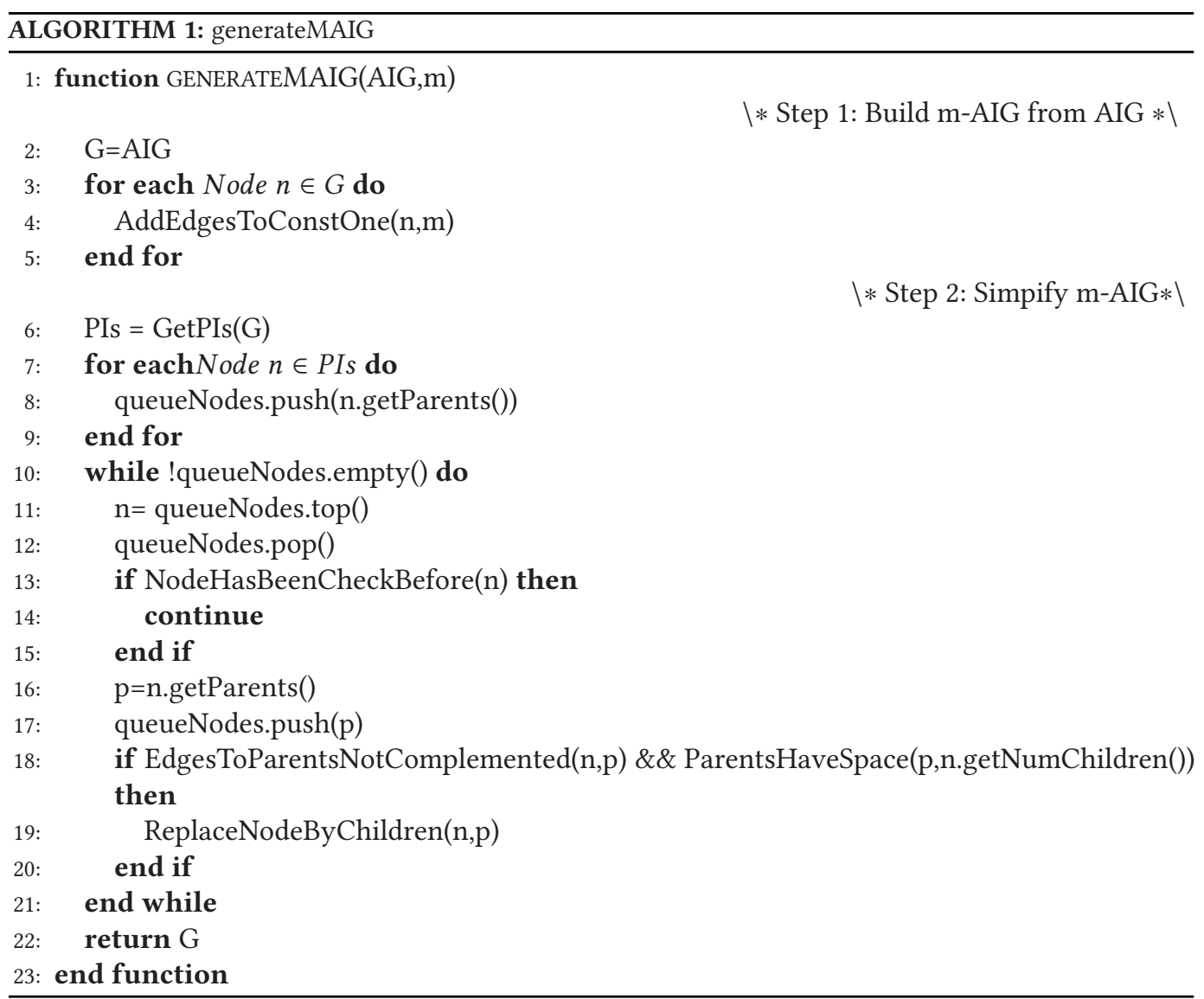

since $\overline{A N D(a, b)} \neq A N D(\bar{a}, \bar{b})$. The m-AIG in Figure 15(b) cannot be simplified, since the parent $p_{2}$ cannot have additional edges to all the children of $n_{1}$.

The process of generating and simplifying m-AIGs is summarized in Algorithm 1. The first step of generating the m-AIG from the given AIG can be seen in Lines 2-5. The AIG is copied into the mAIG graph $G$ and $m-2$ inputs are added to each node and connected to the constant 1 . Subsequently, Lines 6-21 show the simplification process. Starting from the parents PIs, all nodes are checked exactly once. Note that the PIs are not checked, since they cannot be removed from the graph. To ensure that each node is used exactly once, a hash map can be used to realize the function in Line 13. The conditions for removing a node $n$ are checked in Line 18 . That is, all edges to the parents are not complemented, and the parents have enough edges to the constant 1 , such that they can be redirected to the children of $n$. The node is removed and replaced by its children in Line 19. Finally, after the graph has been simplified, it is returned in Line 22.

Since m-AIGs are a super class of classical AIGs, they cannot be canonical. However, AIGs can be functionally reduced in such a way, that no two nodes in the AIG compute the same function in the terms of the PIs. This is called semi-canocity [14]. By using our generation algorithm, the function implemented by each node that is not merged in the second step is not changed in terms of the PIs. Thus, if the initial AIG was semi-canonical, then the generated m-AIG will be, too. 


\subsection{Mapping of $\mathrm{m}$-AIGto ReRAM Crossbar}

m-AIGs are especially suited for synthesis of Boolean functions on ReRAM, since they are compact (at most as many nodes as the corresponding AIG) and can be efficiently mapped to ReRAM devices. In fact, existing synthesis schemes for AIGs, such as the one proposed in Section 4.3, can be adapted for m-AIGs.

Using the approach proposed in Section 4.3, to compute an m-AIG node, one of the inputs has to be used as host operand. Again, we propose to use inversion for initialization. After initialization, if an m-AIG node has $k \leq m$ non-constant children, then we have to perform $k-1 \mathrm{RM}_{3}$ additional computations. However, since we want to compute multiple nodes of each level in parallel this may lead to an overhead, since each node may have a different number of non-constant children. If this is the case, then we have to wait for the computation of the node with the most number of non-constant children to finish before we can use the computed values. To minimize this overhead, we propose to sort the nodes by the number of their non-constant children before the computation. Since the structure of the graph is known before the computation, this can be done offline and thus without any additional cost.

\section{SIMULATION RESULTS}

In this section, our simulation results are presented. Our simulations are seperated in three parts. In the first part, we perform simulations for our proposed BDD synthesis approach. Subsequently, in the second part we compare our proposed synthesis approaches for AIGs to the state of the art. Finally, we compare m-AIGs to both AIGs and BDDs and handcrafted adder designs.

\subsection{BDDs}

We have implemented the proposed approach using CUDD 3.0.0 [20]. We compare our results to those presented in Reference [6] and Reference [16], which were optimized for the number of operations in a BDD-based implementation. To have comparable results, we have chosen benchmarks from the same benchmark set (23 circuits from LGSynth91 [24]) and the same parameters for the EA as the authors of Reference [16]. We have applied the EA in five independent runs to each benchmark and evaluated the best of them, i.e., the smallest number of operations. We assumed the register size to be 16 bit.

Table 1 shows the results for the best of the five runs. The first four columns show the characteristics of the benchmarks. The first column shows the name of the respective circuit, the second column the number of PIs and outputs, and columns 3 and 4 the number of devices $(R)$ and operations $(O P)$ needed if the natural variable ordering is applied and the proposed synthesis approach is used. The fifth and sixth columns show the results of Reference [6], where again $R$ is the number of ReRAM devices needed and $O P$ is the number of operations. Next, the results of Reference [16] are shown (columns 7 to 10). First, columns 7 and 8 show the results for an Material Implication(IMP) based implementation of the BDD nodes, while columns 9 and 10 show the results if $\mathrm{RM}_{3}$ is used. Finally, columns 11 and 12 show the absolute results of the EA, while columns 13 and 14 show the reduction relative to the initial results where the natural variable ordering is used in percentages.

We can see that our proposed approach significantly reduces the number of devices and operations needed compared to Reference [6] and Reference [16]. Compared to Reference [6], it needs almost the same number of devices but reduces the number of operations by about 226.4 (which is a reduction of about 66\%) on average. Compared to Reference [16], our proposed approach needs 37.6 (8\%) fewer devices if IMP is used and 93.7 (17\%) fewer devices if $\mathrm{RM}_{3}$ is used for the implementation of Reference [16]. Further, on average, our approach uses 105.0 (47\%) 
Table 1. Comparison of Results to Chakraborti et al. [6] and Shirinzadeh et al. [16]

\begin{tabular}{|c|c|c|c|c|c|c|c|c|c|c|c|c|c|}
\hline \multirow[b]{3}{*}{ Name } & \multirow[b]{3}{*}{$\mathrm{PI} / \mathrm{PO}$} & \multirow[b]{3}{*}{$\mathrm{R}$} & \multirow[b]{3}{*}{$\mathrm{OP}$} & \multirow{2}{*}{\multicolumn{2}{|c|}{$\begin{array}{c}\text { Chakraborti } \\
\text { et al. [6] }\end{array}$}} & \multicolumn{4}{|c|}{ Shirinzadeh et al. [16] } & \multirow{2}{*}{\multicolumn{4}{|c|}{ Proposed Approach }} \\
\hline & & & & & & \multicolumn{2}{|c|}{ IMP } & \multicolumn{2}{|c|}{$\mathrm{RM}_{3}$} & & & & \\
\hline & & & & $R$ & $\overline{O P}$ & $R$ & $O P$ & $R$ & $O P$ & $R$ & $O P$ & Red R & Red OP \\
\hline 5xp1_90 & 10 & 112 & 19 & 84 & 73 & 65 & 42 & 62 & 4 & 32 & 14 & $71.4 \%$ & $3 \%$ \\
\hline & & & 180 & 642 & 334 & 1,014 & 77 & 1,069 & & & 94 & & \\
\hline apex1 & $45 / 45$ & 8,016 & 1,177 & 1,626 & 705 & 3,040 & 277 & 3,185 & 232 & 1,056 & 244 & & \\
\hline apex 4 & $9 / 19$ & 912 & 147 & 2,073 & 447 & 2,224 & 62 & 2,588 & 53 & 720 & 123 & & \\
\hline ap & $135 / 99$ & 3,600 & 621 & 770 & 1,169 & 220 & 813 & 235 & 678 & 1,760 & 390 & & $.2 \%$ \\
\hline apex7 & $49 / 37$ & 2,176 & 358 & 290 & 437 & 190 & 328 & 151 & 279 & 512 & 130 & $76.5 \%$ & $3.7 \%$ \\
\hline b9 & $41 / 21$ & 496 & 115 & 125 & 298 & 77 & 267 & 92 & 226 & 240 & 95 & $51.6 \%$ & $17.4 \%$ \\
\hline clip & $9 / 5$ & 224 & 42 & 120 & 80 & 93 & 63 & 107 & 54 & 64 & 20 & $71.4 \%$ & $52.4 \%$ \\
\hline cm150 & $21 / 1$ & 130,848 & 16,412 & 56 & 127 & 28 & 127 & 30 & 10 & 96 & 46 & $99.9 \%$ & $99.7 \%$ \\
\hline $\mathrm{Cr}$ & $14 / 5$ & 176 & 37 & 46 & 10 & 38 & 89 & 43 & 7 & 80 & 31 & $54.5 \%$ & $16.2 \%$ \\
\hline & $16 / 5$ & 192 & 42 & 42 & 11 & 31 & 108 & 32 & 92 & 80 & 35 & $58.3 \%$ & $16.7 \%$ \\
\hline cordic & $23 / 2$ & 64 & 48 & 32 & 149 & 26 & 140 & 29 & 117 & 64 & 48 & $0 \%$ & $0 \%$ \\
\hline misex1 & $8 / 7$ & 80 & 19 & 83 & 69 & 79 & 50 & 91 & 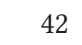 & 48 & 17 & $40.0 \%$ & $10.5 \%$ \\
\hline $\mathrm{mi}$ & $14 / 14$ & 528 & 185 & 444 & 1 & 681 & 86 & 781 & 7 & 304 & 83 & $42.4 \%$ & $55.1 \%$ \\
\hline parity & & 32 & 32 & 23 & 11 & 6 & 112 & 7 & ( & 32 & 32 & $0 \%$ & $0 \%$ \\
\hline seq & $41 / 35$ & 7,040 & 19,099 & 1,566 & 692 & 1,207 & 248 & 1,398 & 207 & 848 & 231 & $98.5 \%$ & $98.8 \%$ \\
\hline $\mathrm{t} 48$ & $16 / 1$ & 144 & 39 & 26 & 10 & 16 & 100 & 30 & 84 & 144 & 39 & $0 \%$ & $0 \%$ \\
\hline tables & $17 / 15$ & 344 & 390 & 580 & 16 & 1,346 & 107 & 1,511 & 90 & 336 & 105 & $75.0 \%$ & $73.1 \%$ \\
\hline too_larg & & $0<0$ & 096 & 282 & 23 & 182 & 229 & 212 & & 384 & 114 & $85.4 \%$ & $\%$ \\
\hline$\times 1$ & $51 / 3$ & 1,408 & 292 & 230 & 39 & 186 & 333 & 217 & 282 & 688 & 159 & $51.1 \%$ & $45.5 \%$ \\
\hline$\times 2$ & & & 29 & 60 & 80 & 45 & 65 & 52 & 55 & 48 & 21 & $66.6 \%$ & $27.5 \%$ \\
\hline$\times 3$ & $135 / 9$ & 3,2 & 71 & 770 & 1,169 & 215 & 813 & 252 & 678 & 1,744 & 389 & $47.1 \%$ & $45.7 \%$ \\
\hline$\times 4$ & $94 / 71$ & 1,968 & 363 & 401 & 642 & 209 & 573 & 333 & 479 & 720 & 233 & $63.4 \%$ & $35.8 \%$ \\
\hline$\overline{\mathrm{AVC}}$ & & 03.8 & 8.0 & 0.9 & 3.5 & 37.7 & 2.1 & 543.8 & 37.3 & 50.1 & 17.1 & $55.3 \%$ & 4 \\
\hline
\end{tabular}

$P I / P O$ : number of PIs/number of primary outputs.

$R$ : number of ReRAM devices, $O P$ : number of operations.

Red: Reduction achieved, relative to naive implementation.

and $70.2(37 \%)$ fewer operations if IMP or $\mathrm{RM}_{3}$ are used for the implementation of Reference [16], respectively.

\subsection{AIGs}

All results shown use wordsize $r=16$ and hole parameter $h=12$, unless stated otherwise. We evaluate instruction sets generated our approach in terms of delay and area. Delay $(D)$ is expressed as the total number of array accesses, which may be computations $(C)$ or reads $(R)$. Area is expressed as total number of ReRAM devices needed by the program $(Z)$. We compare our results with ReVAMP [2] as well as with a Staircase-based synthesis approach using MAGIC [25]. Subscripts are used to indicate which approach produced a result, where $p$ represents our proposed approach, $r$ represents ReVAMP, and $m$ represents the synthesis approach using MAGIC. To ensure a fair comparison we report $D_{p}^{*}$, which is the total delay produced by our proposed approach increased by the worst-case input arrangement cost discussed in Section 4.3.4.

6.2.1 Comparison with ReVAMP. We evaluate our proposed approach on benchmarks provided by EPFL ${ }^{1}$ and compare with ReVAMP that ran the same benchmarks in Reference [2]. Table 2 shows

\footnotetext{
${ }^{1}$ http://lsi.epfl.ch/mig.
} 
Table 2. Comparison with ReVAMP

\begin{tabular}{|c|c|c|c|c|c|c|c|c|c|c|c|c|}
\hline Benchmark & $C_{p}$ & $C_{r}$ & Speedup & $R_{p}$ & $R_{r}$ & Speedup & $D_{p}^{*}$ & $D_{r}$ & Speedup & $Z_{p}$ & $Z_{r}$ & Improv. \\
\hline pci_spoci_ctrl & 300 & 1,523 & $507 \%$ & 868 & 1,328 & $152 \%$ & 1,168 & 2,851 & $222 \%$ & 656 & 1,360 & $207 \%$ \\
\hline revx & 2,639 & 14,575 & $552 \%$ & 7,366 & 14,004 & $190 \%$ & 10,005 & 28,579 & $284 \%$ & 512 & 9,776 & $1909 \%$ \\
\hline sqrt32 & 1,414 & 4,216 & $298 \%$ & 1,785 & 3,948 & $221 \%$ & 3,199 & 8,164 & $251 \%$ & 240 & 2,720 & $1133 \%$ \\
\hline square & 5,125 & 30,988 & $604 \%$ & 17,472 & 29,880 & $171 \%$ & 22,597 & 60,868 & $268 \%$ & 6,992 & 23,264 & $332 \%$ \\
\hline des_area & 1,356 & 5,971 & $440 \%$ & 2,875 & 5,639 & $196 \%$ & 4,231 & 11,610 & $246 \%$ & 1,904 & 4,880 & $256 \%$ \\
\hline ac97_ctrl & 2,572 & 8,803 & $342 \%$ & 5,579 & 7,520 & $134 \%$ & 8,151 & 16,323 & $146 \%$ & 7,392 & 14,928 & $201 \%$ \\
\hline hamming & 986 & 3,603 & $365 \%$ & 2,205 & 3,450 & $156 \%$ & 3,191 & 7,053 & $204 \%$ & 624 & 2,880 & $461 \%$ \\
\hline tv80 & 2,245 & 11,219 & $499 \%$ & 6,696 & 10368 & $154 \%$ & 8,941 & 21,587 & $228 \%$ & 2,912 & 9,248 & $317 \%$ \\
\hline mem_ctrl & 2,698 & 9,497 & $352 \%$ & 7,709 & 8,405 & $109 \%$ & 10,407 & 17,902 & $149 \%$ & 4,128 & 10,000 & $242 \%$ \\
\hline i2c & 306 & 1,450 & $473 \%$ & 754 & 1,247 & $165 \%$ & 1,060 & 2,697 & $214 \%$ & 768 & 1,328 & $172 \%$ \\
\hline ss_pcm & 114 & 313 & $274 \%$ & 151 & 257 & $170 \%$ & 265 & 570 & $140 \%$ & 336 & 496 & $147 \%$ \\
\hline usb_phy & 118 & 538 & $455 \%$ & 303 & 460 & $151 \%$ & 421 & 998 & $173 \%$ & 400 & 592 & $148 \%$ \\
\hline $\max$ & 1,209 & 4,431 & $366 \%$ & 1,527 & 4,184 & $274 \%$ & 2,736 & 8,615 & $252 \%$ & 1,536 & 5,024 & $327 \%$ \\
\hline spi & 928 & 4,615 & $497 \%$ & 1,960 & 4,301 & $219 \%$ & 2,888 & 8,916 & $274 \%$ & 1,504 & 4,272 & $284 \%$ \\
\hline sasc & 183 & 602 & $328 \%$ & 350 & 514 & $146 \%$ & 533 & 1,116 & $156 \%$ & 560 & 912 & $162 \%$ \\
\hline $\operatorname{div} 16$ & 1,854 & 8,375 & $451 \%$ & 4,741 & 7,825 & $165 \%$ & 6,595 & 16,200 & $244 \%$ & 544 & 5,472 & $1005 \%$ \\
\hline MAC32 & 2,642 & 15,980 & $604 \%$ & 8,190 & 15,363 & $187 \%$ & 10,832 & 31,343 & $286 \%$ & 3,600 & 12,544 & $348 \%$ \\
\hline pci_bridge32 & 4,393 & 23,826 & $542 \%$ & 11,225 & 21,914 & $195 \%$ & 15,618 & 45,740 & $226 \%$ & 9,440 & 24,736 & $262 \%$ \\
\hline MŪL32 & 2,459 & 14,047 & $571 \%$ & 6,220 & 13,389 & $215 \%$ & 8,679 & 27,436 & $313 \%$ & 2,384 & 12,496 & $524 \%$ \\
\hline systemcdes & 712 & 3,312 & $465 \%$ & 1,826 & 3,090 & $169 \%$ & 2,538 & 6,402 & $216 \%$ & 1,072 & 3,120 & $291 \%$ \\
\hline comp & 6,703 & 32297 & $481 \%$ & 17,450 & 31,293 & $179 \%$ & 24,153 & 63,590 & $259 \%$ & 5,600 & 18,912 & $337 \%$ \\
\hline systemcaes & 2,742 & 11,100 & $404 \%$ & 5,836 & 10,229 & $175 \%$ & 8,578 & 21,329 & $217 \%$ & 4,720 & 11,536 & $244 \%$ \\
\hline simple_spi & 227 & 930 & $409 \%$ & 534 & 794 & $148 \%$ & 761 & 1,724 & $179 \%$ & 560 & 1,200 & $214 \%$ \\
\hline usb_funct & 3,719 & 16,054 & $431 \%$ & 11,649 & 14,269 & $122 \%$ & 15,368 & 30,323 & $170 \%$ & 7,840 & 16,912 & $215 \%$ \\
\hline AVG & 1,986 & 9511 & $479 \%$ & 5,220 & 8,903 & $170 \%$ & 172,915 & 18,414 & $232 \%$ & 2,759 & 8,275 & $299 \%$ \\
\hline
\end{tabular}

the results. AIG versions were included in the benchmarks and could be readily used for instruction generation with our proposed approach. We achieve a significant speedup of factor 4.79 regarding computations and a speedup of 1.70 regarding reads. Taking input placement into account, the solution generated by our proposed approach is faster by a factor of 2.32 while at the same time taking only about a third of the area. It is worth noting that while ReVAMP does take the cost for arranging inputs on the array into account, it does not account for the time it takes to read these values in the first place. These results further corroborate that the MIG, which is used by the ReVAMP synthesis as logic representation, fails to enable the parallel computational power that is provided by ReRAM array computing. Moreover, we see that the dynamic ReRAM allocation used by our proposed approach provides significant area benefits over the static allocation used by ReVAMP.

6.2.2 Comparison with Staircase Synthesis Using MAGIC. The results of evaluating our proposed approach on the combinational functions of the ISCAS benchmark suite [5] are shown in Table 3. The benchmarks were optimized with ABC [4] using the command sequence if - ; iresyn before instruction generation. We compare our proposed approach with a state-of-the-art synthesis approach using MAGIC [25]. Each MAGIC operation takes two array accesses; one for initializing the output device and one for the actual computation. We report the total delay $D_{m}$ in terms of array accesses. We can observe a speedup of factor 2.13 while using fewer than $\frac{1}{6}$ of the number of devices. This ratio only considers the ReRAM devices taking part in the computation. However, the Staircase synthesis has to allocate an area that can be more than an order of magnitude larger than the number of utilized ReRAM devices to compute the benchmarks [25]. Furthermore, it does not account for the cost of arranging inputs in the exact needed place on the array.

6.2.3 Scalability. Figure 16 compares the performance of our proposed approach for different wordsizes $r$ and hole parameters $h$. The numbers shown are total delay $D_{c}^{*}$ and total number of 
Table 3. Staircase Comparison

\begin{tabular}{lrrrrrr}
\hline Benchmark & $D_{p}^{*}$ & $D_{m}$ & Speedup & $Z_{p}$ & $Z_{m}$ & Improv. \\
\hline c2670 & 955 & 1,102 & $115 \%$ & 464 & 1,513 & $326 \%$ \\
c6288 & 2,521 & 7,502 & $297 \%$ & 544 & 6,369 & $1170 \%$ \\
c1355 & 311 & 472 & $151 \%$ & 192 & 1,035 & $539 \%$ \\
c7552 & 2,002 & 4,364 & $217 \%$ & 720 & 4,461 & $619 \%$ \\
c880 & 446 & 854 & $191 \%$ & 208 & 889 & $427 \%$ \\
c3540 & 1,090 & 2,870 & $263 \%$ & 416 & 2,764 & $664 \%$ \\
c499 & 311 & 484 & $155 \%$ & 192 & 1,021 & $531 \%$ \\
c1980 & 449 & 1,034 & $230 \%$ & 192 & 1,087 & $566 \%$ \\
c432 & 306 & 450 & $147 \%$ & 112 & 405 & $361 \%$ \\
c5315 & 1,831 & 2,722 & $148 \%$ & 640 & 3,553 & $555 \%$ \\
\hline AVG & 1,022 & 2,185 & $213 \%$ & 368 & 2,310 & $627 \%$ \\
\hline
\end{tabular}

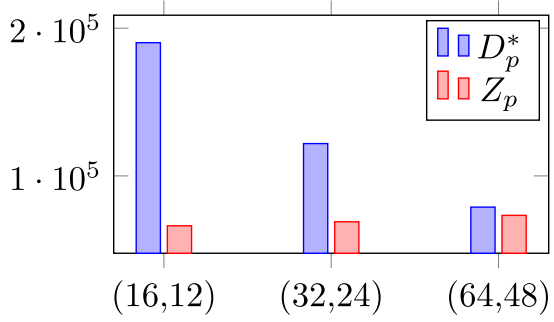

Fig. 16. Performance of our proposed approach for different values of $(r, h)$.

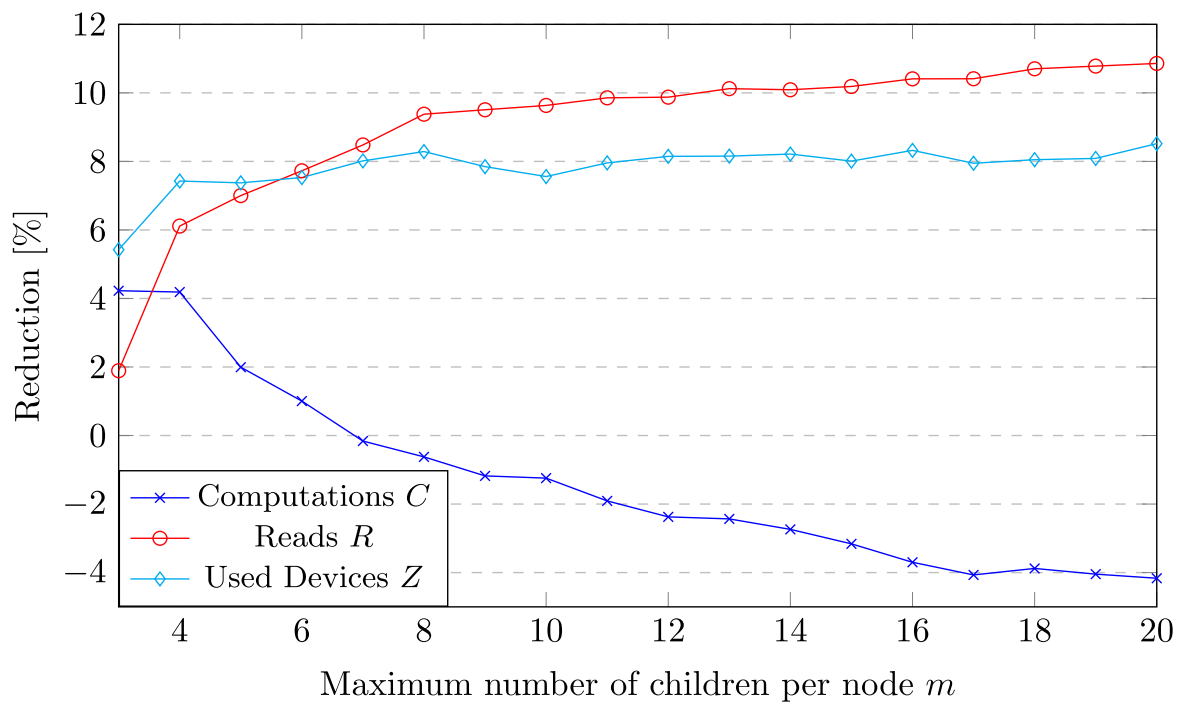

Fig. 17. Average reduction for different values of $m$.

ReRAM devices $Z_{c}$ needed for all benchmarks of Table 2 combined. The results show that our proposed approach can be scaled up to achieve additional speedup, while the necessary number of ReRAM devices changes only insignificantly. All compilations ran in less than half a second per benchmark on an Intel Core i7-8550U processor with 24 GB of RAM.

\section{3 m-AIGs}

In this section, we describe the simulation results for our proposed m-AIGs. We have used the same benchmarks as in Section 6.2 and use the results for AIGs as baseline. For comparability, we have used 16 as wordsize $r$ and 12 as hole paramter $h$. To evaluate the benefit of using m-AIGs, we increase the maximum number of non-constant children step by step and calculate the resulting number of reads $R$, computations $C$, and used devices $Z$ for each function. In addition, we also show a comparison of our m-AIG-based appraoch to handcrafted designs.

6.3.1 Influence of $m$ on the Results. The average over all simulations can be seen in Figure 17 . The red line shows the number of reads, the blue line the delay, and the cyan line the number of used devices. We have plotted the reduction in percentages compared to the results from Section 6.2 
over the maximum number of children per node. A positive reduction means, that the number has decreased, while a negative reduction indicates an actual increase of the corresponding measure.

We can see that, for the maximum number of children $m \in\{3,4\}$ the number of computations $C$, as well as the number of reads $R$ and area $Z$ decreases. However, while for $m \geq 5$ the number of reads $R$ and the used area $Z$ decrease further, the number of computations $C$ actually starts to increase, surpassing the original value at $m=7$. The decrease in reads $R$ saturates at around $10 \%$, while the decrease in area $Z$ reaches up to $8 \%$. The number of computations $z$ are increased by up to $4 \%$ on average. Note that for AIGs the absolute number of reads $R$ are a lot higher than the number of computations $C$ (cf. Table 2), and thus the overall number of operations on the array decreases for all $m$. For all metrics, computations $C$, reads $R$, and area $Z$, we can see that they all almost stop to change at some point in the graph. This is because increasing $m$ does not necessarily change the m-AIG besides edges to constant 1 if no nodes can be merged or only small changes can be made (see Section 5.2).

Since we have fewer nodes, it is clear that the area $Z$ and number of reads $R$ decreases on average the higher $m$ is chosen. Fewer intermediate values need to be stored and read. However, the increase in instructions might be surprising at first. Recall the mapping procedure presented in Section 5.3. Since multiple nodes are computed in parallel and $k-1 \mathrm{RM}_{3}$ operations need to be performed, where $k \leq m$ is the maximum number of non-constant children of the nodes, which are currently computed in parallel. Thus, it is clear that if we have only a small number of nodes with a lot of children on each level, then the number of computations increases.

6.3.2 Detailed Analysis. From the results in Section 6.3.1, we can see that for m-AIGs, $m \in$ $\{3,4\}$ gives the smallest number of instructions on average, while for large $m$ the number of reads and area is most reduced. Thus, one can choose $m$ according to the technology, where the access times and wear out for reads and instructions may differ and the given number of ReRAM devices may be limited. The gives m-AIGs the versatility to be adjusted by the designer for their concrete application.

Detailed results can be seen in Table 4. Columns 2-4 show the number of instructions $C$ for AIGs and m-AIGs with $m=4$ and $m=20$, respectively. Columns 5-7 and 8-10 show the results for the reads $R$ and used ReRAM devices $Z$.

We can see that, in contrast to the averages shown in Section 6.3.1 for some benchmarks, the number of instructions actually decreases for larger values of $m$. Consider, for example, the benchmark sysemcaes. Here, the number of instructions $C$ for $m=4$ is smaller than for AIGs and for $m=20$ the value decreases even more. However, for a small number of benchmarks, the number of reads $R$ for m-AIGs increases compared to AIGs (e.g., MUL32). When converting AIGs to $\mathrm{m}$-AIGs, nodes are merged and sorted with respect to the number of non-constant children. This changes the order in which the nodes are computed, which may result in different hole sizes during the computation of the function. For some borderline cases, this might increase the overall number of read words, which can also be seen in these results. However, compared to the total number of simulations, the number of these cases is rather small.

6.3.3 Comparison to Handcrafted Designs. For in-memory computing, a lot of handcrafted designs are available. In this section, we show that we can achieve comparable results with our proposed automated synthesis flow by comparing our approach to handcrafted adder designs. Again, we assume the wordsize to be $r=16$ and use $h=12$ as hole parameter.

To compare the handcrafted designs to our proposed m-AIG-based synthesis approach, we use YOSYS [23] to synthesize an 8-bit Ripple-Carry Adder (RCA) to AIG. Using our proposed methodology, for both $m=3$ and $m=20$ we need 80 devices and a delay of 137 in terms of accesses to the array. 
Table 4. Comparison of Results for Different Values of $m$

\begin{tabular}{lrrrrrrrrr}
\hline Benchmark & $C_{A I G}$ & $C_{m=4}$ & $C_{m=20}$ & $R_{A I G}$ & $R_{m=4}$ & $R_{m=20}$ & $Z_{A I G}$ & $Z_{m=4}$ & $Z_{m=20}$ \\
\hline pci_spoci_ctrl & 300 & 298 & 352 & 868 & 769 & 736 & 656 & 656 & 640 \\
revx & 2,639 & 2,827 & 2,971 & 7,366 & 7,135 & 6,941 & 512 & 480 & 464 \\
sqrt32 & 1,414 & 1,401 & 1,401 & 1,785 & 1,639 & 1,639 & 240 & 272 & 272 \\
square & 5,125 & 5,150 & 5,150 & 17,472 & 17,114 & 17,114 & 6,992 & 6,912 & 6,912 \\
des_area & 1,356 & 1,181 & 1,402 & 2,875 & 2,589 & 2,525 & 1,904 & 1,520 & 1,616 \\
ac97_ctrl & 2,572 & 2,529 & 2,571 & 5,579 & 5,881 & 5,635 & 7,392 & 6,640 & 6,816 \\
hamming & 986 & 995 & 1,107 & 2,205 & 2,067 & 2,026 & 624 & 624 & 624 \\
tv80 & 2,245 & 2,137 & 2,412 & 6,696 & 6,175 & 5,621 & 2,912 & 2,688 & 2,576 \\
mem_ctrl & 2,698 & 2,426 & 2,820 & 7,709 & 7,179 & 5,702 & 4,128 & 3,584 & 3,488 \\
i2c & 306 & 289 & 339 & 754 & 627 & 556 & 768 & 672 & 640 \\
ss_pcm & 114 & 107 & 107 & 151 & 142 & 142 & 336 & 304 & 304 \\
usb_phy & 118 & 118 & 122 & 303 & 271 & 262 & 400 & 352 & 336 \\
max & 1,209 & 1,138 & 1,258 & 1,527 & 1,610 & 1,582 & 1,536 & 1,408 & 1,408 \\
spi & 928 & 837 & 864 & 1,960 & 1,757 & 1,497 & 1,504 & 1,392 & 1,360 \\
sasc & 183 & 173 & 170 & 350 & 323 & 305 & 560 & 512 & 512 \\
div16 & 1,854 & 1,874 & 2,050 & 4,741 & 4,428 & 4,162 & 544 & 496 & 464 \\
MAC32 & 2,642 & 2,727 & 2,757 & 8,190 & 8,141 & 8,147 & 3,600 & 3,696 & 3,696 \\
pci_bridge32 & 4,393 & 4,145 & 4,908 & 11,225 & 10,969 & 10,027 & 9,440 & 9,488 & 8,832 \\
MUL32 & 2,459 & 2,413 & 2,549 & 6,220 & 6,647 & 6,549 & 2,384 & 2,208 & 2,208 \\
systemcdes & 712 & 671 & 694 & 1,826 & 1,647 & 1,587 & 1,072 & 864 & 848 \\
comp & 6,703 & 5,369 & 7,455 & 17,450 & 16,604 & 14,380 & 5,600 & 4,384 & 4,112 \\
sysemcaes & 2,742 & 2,407 & 2,396 & 5,836 & 5,448 & 5,335 & 4,720 & 4,544 & 4,576 \\
simple_spi & 227 & 234 & 252 & 534 & 441 & 428 & 560 & 496 & 512 \\
usb_funct & 3,719 & 3,455 & 3,995 & 11,649 & 10,581 & 10,231 & 7,840 & 7,424 & 7,744 \\
\hline & & & & & & & & &
\end{tabular}

In Reference [22], a handcrafted 8-bit adder design was proposed. This adder is designed in an RCA fashion, meaning that the adder consists of several full adders that are connected in a carry chain. However, using in-memory computing, the hardware overhead can be kept low. Overall, the authors propose an IMP-based methodology that only uses 27 ReRAM devices and 184 array accesses. Thus, while we about need $196 \%$ more devices, our delay is about $25.5 \%$ smaller due to the use of parallelism.

In Reference [18], ORNOR logic for ReRAM is proposed. Based on this ORNOR logic, a handcrafted $N$-bit adder scheme is presented. This scheme uses $6(N+1)$ ReRAM devices and $2 N+15$ array accesses. Thus, for an 8-bit adder, 54 ReRAM devices and 31 array accesses would be needed. This specialized scheme can outperform our proposed methodology in both number of devices and computational cycles. However, the approach presented in Reference [18] is a specialized approach for adders, while our approach is a general approach for all Boolean functions.

The authors of Reference [21], present MAGIC gate-based implementations for $\mathrm{N}$ bit addition in ReRAM. The authors present different schemes: two for conventional ReRAM devices (area or latency optimized) and two for transpose ReRAM devices, where the devices can be written and read from both sides (i.e., from the bitlines and the wordlines). Here, an 8-bit RCA is used as a case study. The presented schemes use

- Conventional Memory, area optimized: 120 array accesses and 30 devices

- Conventional Memory, latency optimized: 97 array accesses and 112 devices 
- Transpose Memory, scheme-1: 121 array accesses and 198 devices

- Transpose Memory, scheme-2: 83 array accesses and 126 devices

including 16 input and 9 output devices. We can see, that the delay of the output of our proposed approach is slightly larger than the handcrafted designs of Reference [21]. However, our proposed approach needs fewer devices than almost all of the implementations schemes in Reference [21]. Only the area optimized computation scheme needs fewer devices than our proposed automated synthesis approach.

Overall, we can say that, while our general approach can be outperformed by specialized synthesis, handcrafted strategies, it is often not by a large margin.

\section{CONCLUSION}

In this article, we have presented schemes for the parallel computation BDD and AIG nodes on ReRAM. First, we have shown how to efficiently perform parallel computation of BDD nodes using the MAC computation capabilities of ReRAM. Further, we have presented a parallel computation scheme for AIG-based synthesis. We have also introduced a new graph representation for Boolean functions called m-AIGs and shown how to generate and compute them efficiently.

In the simulations, we have compared our BDD and AIG synthesis strategies to the state of the art. We have shown that we outperform them in terms of both area and operations. Subsequently, we have shown the efficiency of m-AIGs by comparing them to AIGs. We have shown that for small values of $m$, m-AIGs outperform AIGs in every aspect. However, for large values of $m$, the number of instructions increases, while the number of reads and used devices decreases on average, giving the designer a tuning knob for the optimization with respect to the used application and technology. Finally, we have given a comparison of our m-AIG-based synthesis scheme to handcrafted adder designs for in-memory computing. We have shown that we can compete with handcrafted designs with respect to the delay and area.

\section{REFERENCES}

[1] L. Amarú, P.-E. Gaillardon, and G. De Micheli. 2014. Majority-Inverter Graph: A novel data-structure and algorithms for efficient logic optimization. In Proceedings of the Design Automation Conference (DAC'14). 1-6.

[2] D. Bhattacharjee, Y. Tavva, A. Easwaran, and A. Chattopadhyay. 2020. Crossbar-constrained technology mapping for ReRAM based in-memory computing. IEEE Trans. Comput. 69, 5 (2020), 734-748.

[3] Julien Borghetti, Gregory S. Snider, Philip Kuekes, J. Joshua Yang, Duncan R. Stewart, and R. Stanley Williams. 2010. Memristive switches enable stateful logic operations via material implication. Nature 464, 7290 (2010), 873-876.

[4] Robert Brayton and Alan Mishchenko. 2010. ABC: An academic industrial-strength verification tool. In Proceedings fo the International Conference on Computer Aided Verification. Springer, 24-40.

[5] Franc Brglez. 1985. A neutral netlist of 10 combinatorial benchmark circuits and a target translator in FORTRAN. In Proceedings of the International Symposium on Circuits and Systems, Special Session on ATPG and Fault Simulation. 663-698.

[6] S. Chakraborti, P. V. Chowdhary, K. Datta, and I. Sengupta. 2014. BDD based synthesis of Boolean functions using memristors. In Proceedings of the 9th International Design and Test Symposium (IDT'14). 136-141.

[7] Yi-Chou Chen, C. F. Chen, C. T. Chen, J. Y. Yu, S. Wu, S. L. Lung, and R. Liu and. 2003. An access-transistor-free $(0 \mathrm{~T} / 1 \mathrm{R})$ non-volatile resistance random access memory (RRAM) using a novel threshold switching, self-rectifying chalcogenide device. In Proceedings of the IEEE International Electron Devices Meeting. 37.4.1-37.4.4.

[8] S. Frerix, S. Shirinzadeh, S. Fröhlich, and R. Drechsler. 2019. ComPRIMe: A compiler for parallel and scalable ReRAMbased in-memory computing. In Proceedings of the IEEE/ACM International Symposium on Nanoscale Architectures (NANOARCH'19). 1-6.

[9] S. Fröhlich, S. Shirinzadeh, and R. Drechsler. 2020. Multiply-accumulate enhanced BDD-Based logic synthesis on RRAM crossbars. In Proceedings of the IEEE International Symposium on Circuits and Systems. 1-5.

[10] P.-E. Gaillardon, L. Amarú, A. Siemon, E. Linn, R. Waser, A. Chattopadhyay, and G. De Micheli. 2016. The programmable logic-in-memory (PLiM) computer. In Proceedings of the Design, Automation Test in Europe Conference Exhibition (DATE'16). 427-432. 
[11] R. Ben Hur, N. Wald, N. Talati, and S. Kvatinsky. 2017. Simple magic: Synthesis and in-memory Mapping of logic execution for memristor-aided logic. In Proceedings of the IEEE/ACM International Conference on Computer-Aided Design (ICCAD’17). 225-232.

[12] Andreas Kuehlmann, Viresh Paruthi, Florian Krohm, and Malay K Ganai. 2002. Robust boolean reasoning for equivalence checking and functional property verification. IEEE Trans. Comput.-Aid. Des. Integr. Circ. Syst. 21, 12 (2002), 1377-1394.

[13] E. Linn, R. Rosezin, S. Tappertzhofen, U. Böttger, and R. Waser. 2012. Beyond von Neumann-logic operations in passive crossbar arrays alongside memory operations. Nanotechnology 23, 30 (2012), 305205. https://doi.org/10.1088/ 0957-4484/23/30/305205

[14] Alan Mishchenko, Satrajit Chatterjee, Roland Jiang, and Robert K Brayton. 2005. FRAIGs: A Unifying Representation for Logic Synthesis and Verification. Technical Report. ERL Technical Report.

[15] R. Rosezin, E. Linn, C. Kugeler, R. Bruchhaus, and R. Waser. 2011. Crossbar logic using bipolar and complementary resistive switches. IEEE Electr. Device Lett. 32, 6 (2011), 710-712. https://doi.org/10.1109/LED.2011.2127439

[16] Saeideh Shirinzadeh, Mathias Soeken, Pierre-Emmanuel Gaillardon, and Rolf Drechsler. 2018. Logic synthesis for RRAM-based in-memory computing. IEEE Trans. Comput.-Aid. Des. Integr. Circ. Syst. 37, 7 (2018), 1422-1435.

[17] Saeideh Shirinzadeh, Mathias Soeken, Daniel Große, and Rolf Drechsler. 2017. An adaptive prioritized $\epsilon$-preferred evolutionary algorithm for approximate BDD optimization. In Proceedings of the Genetic and Evolutionary Computation Conference. 1232-1239.

[18] A. Siemon, R. Drabinski, M. J. Schultis, X. Hu, E. Linn, A. Heittmann, R. Waser, D. Querlioz, S. Menzel, and J. S. Friedman. 2019. Stateful three-input logic with memristive switches. Sci. Rep. 9, 1 (2019), 14618. https://doi.org/10. 1038/s41598-019-51039-6

[19] M. Soeken, S. Shirinzadeh, P. Gaillardon, L. G. Amarú, R. Drechsler, and G. De Micheli. 2016. An MIG-based compiler for programmable logic-in-memory architectures. In Proceedings of the 53nd ACM/EDAC/IEEE Design Automation Conference (DAC'16). 1-6.

[20] F. Somenzi. University of Colorado at Boulder, 2015. CUDD: CU decision diagram package-release 3.0.0.

[21] N. Talati, S. Gupta, P. Mane, and S. Kvatinsky. 2016. Logic design within memristive memories using memristor-aided loGIC (MAGIC). IEEE Trans. Nanotechnol. 15, 4 (2016), 635-650. https://doi.org/10.1109/TNANO.2016.2570248

[22] M. Teimoory, A. Amirsoleimani, J. Shamsi, A. Ahmadi, S. Alirezaee, and M. Ahmadi. 2014. Optimized implementation of memristor-based full adder by material implication logic. In Proceedings of the 21st IEEE International Conference on Electronics, Circuits and Systems (ICECS). 562-565. https://doi.org/10.1109/ICECS.2014.7050047

[23] Clifford Wolf. Yosys-Yosys Open Synthesis Suite. Retrieved from http://www.clifford.at/yosys/about.html.

[24] Saeyang Yang. 1991. Logic Synthesis and Optimization Benchmarks User Guide Version 3.0. (1991).

[25] Alwin Zulehner, Kamalika Datta, Indranil Sengupta, and Robert Wille. 2019. A staircase structure for scalable and efficient synthesis of memristor-aided logic. In Proceedings of Asia and South Pacific Design Automation Conference. 237-242.

Received July 2020; revised December 2020; accepted February 2021 